\title{
The Integrator complex cleaves nascent mRNAs to attenuate transcription
}

\author{
Deirdre C. Tatomer, ${ }^{1}$ Nathan D. Elrod ${ }^{2}$ Dongming Liang, ${ }^{1}$ Mei-Sheng Xiao, ${ }^{1}$ Jeffrey Z. Jiang, ${ }^{1}$ \\ Michael Jonathan, ${ }^{1}$ Kai-Lieh Huang, ${ }^{2}$ Eric J. Wagner, ${ }^{2}$ Sara Cherry, ${ }^{3}$ and Jeremy E. Wilusz ${ }^{1}$ \\ ${ }^{1}$ Department of Biochemistry and Biophysics, University of Pennsylvania Perelman School of Medicine, Philadelphia, \\ Pennsylvania 19104, USA; ${ }^{2}$ Department of Biochemistry and Molecular Biology, University of Texas Medical Branch at Galveston, \\ Galveston, Texas 77550, USA; ${ }^{3}$ Department of Microbiology, University of Pennsylvania Perelman School of Medicine, \\ Philadelphia, Pennsylvania 19104, USA
}

Cellular homeostasis requires transcriptional outputs to be coordinated, and many events post-transcription initiation can dictate the levels and functions of mature transcripts. To systematically identify regulators of inducible gene expression, we performed high-throughput RNAi screening of the Drosophila Metallothionein A (MtnA) promoter. This revealed that the Integrator complex, which has a well-established role in $3^{\prime}$ end processing of small nuclear RNAs (snRNAs), attenuates MtnA transcription during copper stress. Integrator complex subunit 11 (IntS11) endonucleolytically cleaves MtnA transcripts, resulting in premature transcription termination and degradation of the nascent RNAs by the RNA exosome, a complex also identified in the screen. Using RNA-seq, we then identified $>400$ additional Drosophila protein-coding genes whose expression increases upon Integrator depletion. We focused on a subset of these genes and confirmed that Integrator is bound to their $5^{\prime}$ ends and negatively regulates their transcription via IntS11 endonuclease activity. Many noncatalytic Integrator subunits, which are largely dispensable for snRNA processing, also have regulatory roles at these protein-coding genes, possibly by controlling Integrator recruitment or RNA polymerase II dynamics. Altogether, our results suggest that attenuation via Integrator cleavage limits production of many full-length mRNAs, allowing precise control of transcription outputs.

[Keywords: transcription termination; RNA polymerase II; Metallothionein A; MtnA; RNA exosome; IntS11]

Supplemental material is available for this article.

Received June 26, 2019; revised version accepted September 9, 2019.

In response to physiological cues, environmental stress, or exposure to pathogens, specific transcriptional programs are induced (for review, see Vihervaara et al. 2018). These responses are often coordinated, rapid, and robust, in part because many metazoan genes are maintained in a poised state with RNA polymerase II (RNAPII) engaged prior to induction (for review, see Mayer et al. 2017; Core and Adelman 2019). In addition to promoter-proximal pausing, there are many regulatory steps post transcription initiation that dictate the characteristics and fate of mature transcripts. For example, alternative splicing and/or $3^{\prime}$ end processing events can lead to the production of multiple isoforms from a single locus, and these transcripts can have distinct stabilities, translation potential, or subcellular localization (for review, see Braunschweig et al. 2013; Tian and Manley 2017).

It is particularly important that genes produce fulllength functional mRNAs and mechanisms such as telescripting, involving U1 snRNP, actively suppress

Corresponding authors: wilusz@pennmedicine.upenn.edu,

cherrys@pennmedicine.upenn.edu

Article published online ahead of print. Article and publication date are online at http://www.genesdev.org/cgi/doi/10.1101/gad.330167.119. premature cleavage and polyadenylation events in eukaryotic cells (Kaida et al. 2010; Berg et al. 2012; Venters et al. 2019). Nevertheless, many promoters are known to generate short unstable RNAs (Kapranov et al. 2007; Xu et al. 2009; Porrua and Libri 2015). This suggests that premature transcription termination may often occur, thereby limiting RNAPII elongation and production of fulllength mRNAs (for review, see Kamieniarz-Gdula and Proudfoot 2019|. Moreover, this process can be regulated (Brannan et al. 2012; Wagschal et al. 2012; Chalamcharla et al. 2015; Chiu et al. 2018). For example, it was recently shown that the cleavage and polyadenylation factor PCF11 stimulates premature termination to attenuate the expression of many transcriptional regulators in human cells (Kamieniarz-Gdula et al. 2019). Potentially deleterious truncated transcripts generated by premature termination are often removed from cells by RNA surveillance mechanisms, including by the RNA exosome (for

(C) 2019 Tatomer et al. This article is distributed exclusively by Cold Spring Harbor Laboratory Press for the first six months after the full-issue publication date (see http://genesdev.cshlp.org/site/misc/terms.xhtml). After six months, it is available under a Creative Commons License (Attribution-NonCommercial 4.0 International), as described at http://creativecommons.org/licenses/by-nc/4.0/. 
review, see Zinder and Lima 2017; Kamieniarz-Gdula and Proudfoot 2019). However, the full repertoire of cellular factors and cofactors that control the metabolic fate of nascent RNAs, especially during the early stages of transcription elongation, is still unknown.

We thus performed an unbiased genome-scale RNAi screen in Drosophila cells to reveal factors that control the output of a model inducible eukaryotic promoter. Transcription of Drosophila Metallothionein A (MtnA), which encodes a metal chelator, is rapidly induced when the intracellular concentration of heavy metals (e.g., copper or cadmium) is increased (Fig. 1A; for review, see Gun- ther et al. 2012). This increase in transcriptional output is dependent on the MTF-1 transcription factor, which relocalizes to the nucleus upon metal stress and binds to the MtnA promoter (Smirnova et al. 2000). Our RNAi screen identified MTF-1 and other known regulators of MtnA transcription (Marr et al. 2006), but also surprisingly identified the Integrator complex as a potent inhibitor of MtnA during copper stress. Integrator harbors an endonuclease that cleaves snRNAs and enhancer RNAs (Baillat et al. 2005; Lai et al. 2015), and we find that Integrator can likewise cleave nascent MtnA transcripts to limit mRNA production. Using RNA-seq, we find
A

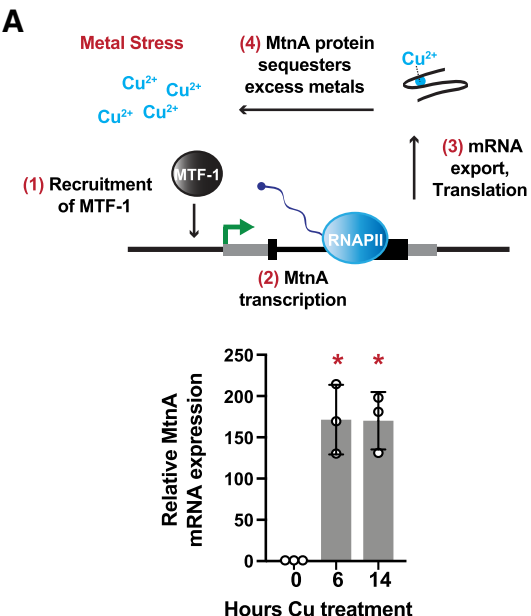

D

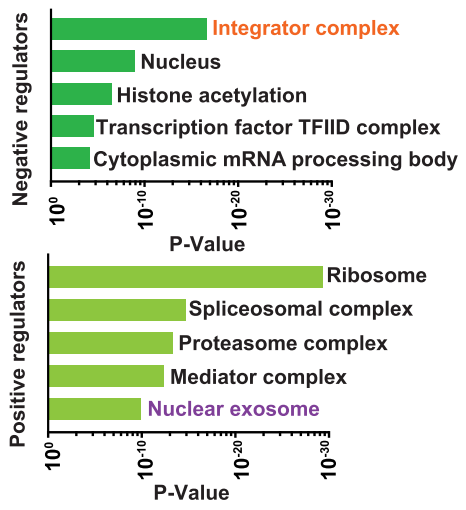

B

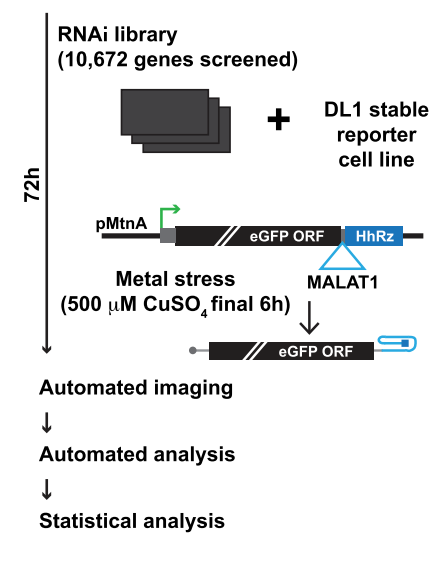

E

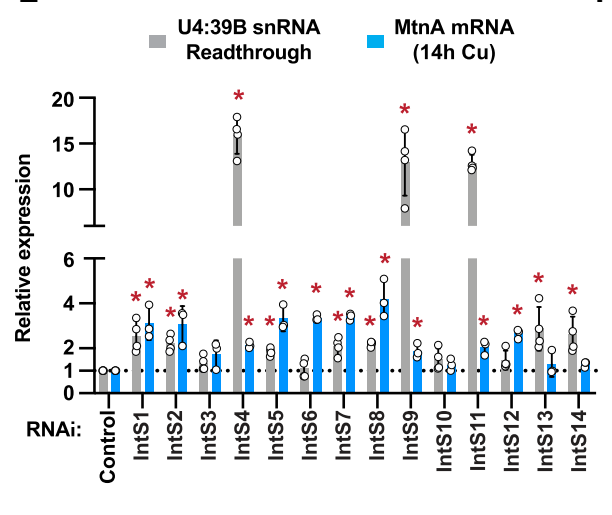

C

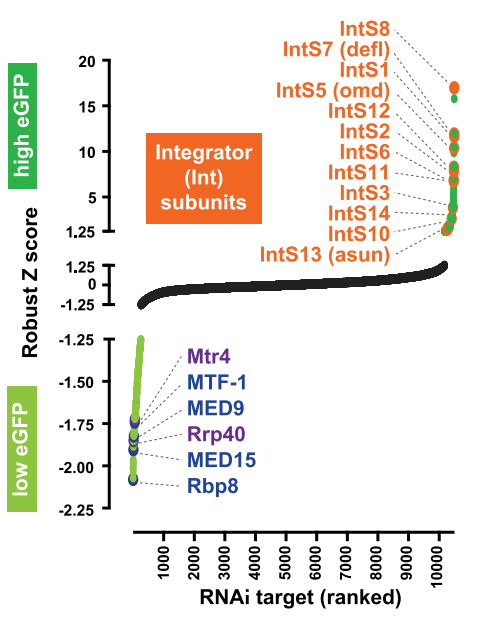

$\mathbf{F}$

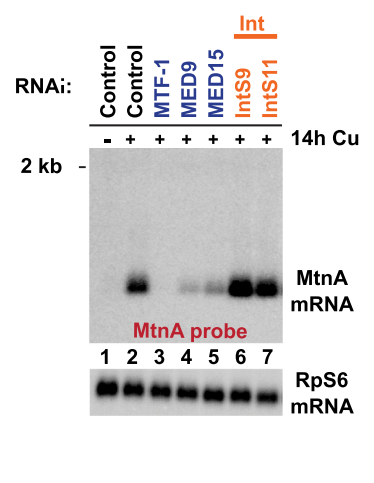

Figure 1. The Integrator complex inhibits expression from the MtnA promoter during copper stress. $(A$, top $)$ Upon metal stress, the transcription factor MTF-1 binds and induces transcription from the MtnA promoter, resulting in production of a protein that sequesters the excess metals to alleviate the stress. (Bottom) Drosophila DL1 cells were treated with $500 \mu \mathrm{M}$ copper sulfate $\left(\mathrm{CuSO}_{4}\right)$ for the indicated times, and RT-qPCR was used to measure endogenous MtnA mRNA expression. Data from three independent experiments were normalized to RpL32 mRNA expression and are shown as mean $\pm \mathrm{SD},\left({ }^{*}\right) P<0.05$. $(B)$ RNAi screen pipeline using DL1 cells stably maintaining an eGFP reporter driven by the MtnA promoter. The self-cleaving hammerhead ribozyme (HhRz) (Dower et al. 2004) generates the eGFP mRNA 3' end, which is then stabilized by the MALAT1 triple helix structure (Wilusz et al. 2012). (C) Robust $Z$-scores of eGFP integrated intensity are shown. RNAi treatments that resulted in increased $(Z$-score $>1.3$, dark green) or decreased $(Z$-score $<-1.3$, light green) eGFP expression are marked, including Integrator subunits (orange), transcription regulators (blue), and RNA exosome components (purple). (D) Gene ontology (GO) analysis was performed to identify categories of genes that are enriched among the negative $(Z$-score $>1.3)$ and positive $(Z$-score $<-1.3)$ regulators of the eGFP reporter. $(E)$ DL1 cells were treated with dsRNAs for $3 \mathrm{~d}$ to induce RNAi and depletion of the indicated factors. Expression of endogenous MtnA mRNA (after $14 \mathrm{~h} \mathrm{CuSO}$ treatment) was quantified by RT-qPCR, and readthrough transcription downstream from the U4:39B snRNA was quantified by northern blotting. Data are shown as mean $\pm \mathrm{SD}, N \geq 3$. $\left({ }^{*}\right) P<0.05$. $(F)$ Representative northern blot of endogenous MtnA mRNA isolated from DL1 cells treated with dsRNA to induce RNAi of the indicated factor. 
hundreds of additional Drosophila protein-coding genes whose expression increases upon Integrator depletion. Focused studies on a subset of these genes confirmed that Integrator can cleave these nascent RNAs, thereby limiting productive transcription elongation. Altogether, we propose that Integrator-catalyzed premature termination can function as a widespread and potent mechanism to attenuate expression of protein-coding genes.

\section{Results}

Genome-scale RNAi screening reveals the Integrator complex as a potent inhibitor of the MtnA promoter during copper stress

To identify regulators of an archetype inducible transcription program, the Drosophila MtnA promoter was cloned upstream of an intronless, non-polyadenylated eGFP reporter (Fig. 1B). Maturation of this mRNA is independent of many canonical mRNA processing events, and we thus reasoned that high-throughput RNAi screening using this reporter should primarily identify transcriptional and translational regulators. Drosophila DL1 cells stably maintaining the reporter were treated with double-stranded RNAs (dsRNAs) for $3 \mathrm{~d}$ and copper was added for the final $6 \mathrm{~h}$ to activate the MtnA promoter and eGFP expression (Fig. 1B). Automated microscopy and image analysis was then used to quantify eGFP fluorescence. A total of 232 factors were required for eGFP expression during copper stress, including ribosomal subunits and well-characterized transcriptional regulators such as RNAPII, Mediator subunits (e.g., MED9 and MED15), and the MTF-1 transcription factor (Fig. 1C,D; Supplemental Table S1; Marr et al. 2006; Günther et al. 2012). Unexpectedly, nearly all 14 subunits of the Integrator (Int) complex (for review, see Baillat and Wagner 2015) were among the most potent negative regulators identified (Fig. 1C,D). Of the $>10,000$ genes screened, depletion of the IntS8 subunit resulted in the largest increase in eGFP expression.

RT-qPCR and western blotting confirmed that the dsRNAs resulted in depletion of the Integrator subunits (Supplemental Fig. S1) and increases in eGFP reporter expression at both the protein (Supplemental Fig. S2A-C) and mRNA levels (Supplemental Fig. S2D,E). These increases in expression upon Integrator depletion were observed regardless of the ORF downstream from the MtnA promoter, as we also observed an increase when eGFP was replaced with nano-Luciferase (Supplemental Fig. $\mathrm{S} 2 \mathrm{~F})$. Likewise, the increases in expression were not dependent on the mRNA $3^{\prime}$ end processing signals downstream from the ORF, as similar increases were observed when a canonical polyadenylation signal was present (Supplemental Fig. S2A-C).

The Integrator complex is present at the endogenous $M t n A$ locus during copper stress and represses $M t n A$ pre-mRNA levels

The Integrator complex has been implicated in a myriad of diseases, interacts with RNAPII, and contains the IntS11
RNA endonuclease that generates the $3^{\prime}$ ends of spliceosomal snRNAs (Baillat et al. 2005; Baillat and Wagner 2015). We confirmed that depleting subunits of the Integrator cleavage module (IntS4, IntS9, or IntS11) resulted in increased snRNA readthrough transcription (Fig. 1E; Supplemental Fig. S3A; Ezzeddine et al. 2011; Albrecht et al. 2018). Nevertheless, because mature snRNAs have long half-lives (Fury and Zieve 1996), their levels only marginally decreased over the $72 \mathrm{~h}$ time course of the experiment (Supplemental Fig. S3B).

Integrator has also been implicated in transcription regulation at enhancers and at a subset of EGF-responsive mRNAs (Gardini et al. 2014; Lai et al. 2015), so we hypothesized that the Integrator complex could be directly acting at the MtnA promoter. Indeed, depletion of Integrator subunits resulted in increased expression of the endogenous MtnA mRNA (Fig. 1E,F; Supplemental Fig. S3C) and premRNA (Fig. 2A) to a similar extent during copper stress. Transcription of the MtnA locus is also induced by cadmium stress (Fig. 2B), but we found that depletion of Integrator subunits strikingly had no effect on MtnA pre-mRNA levels under these conditions (Fig. 2C). This indicates that the Integrator complex can regulate the output of a protein-coding gene in a context-specific manner.

To explore the underlying basis for this distinct regulation of MtnA expression, we used chromatin immunoprecipitation (ChIP)-qPCR to examine the recruitment of Integrator subunits to the MtnA locus upon copper or cadmium stress. We found that IntS1 and IntS12 were recruited to the endogenous MtnA locus upon copper stress (especially to the $5^{\prime}$ end), but their recruitment was significantly less robust during cadmium stress (Fig. 2D). These results suggest that Integrator-mediated repression of MtnA during copper stress is due to Integrator recruitment to the chromatin eliciting direct effects on transcription.

\section{The IntS11 endonuclease activity is required for Integrator-dependent regulation of MtnA expression}

To define how Integrator functions at MtnA during copper stress, we first addressed whether the RNA endonuclease activity of IntS11 is required. The endogenous IntS11 protein was depleted using a dsRNA targeting either the IntS11 ORF or 3' untranslated region (UTR) (Fig. 3A), and this resulted in increased expression of endogenous MtnA mRNA relative to treatment with a control dsRNA (Fig. 3B, lanes 1-3). Expression of a wild-type (WT) IntS11 transgene (Fig. 3A) in cells treated with the IntS11 3' UTR dsRNA restored MtnA expression to levels similar to control treated cells (Fig. 3B, lane 4 vs. 6), whereas expression of a catalytically dead (E203Q) (Baillat et al. 2005) IntS11 transgene did not (Fig. 3B, lane 7 vs. 9). As a control, we confirmed that increased MtnA expression was observed when cells were treated with a dsRNA targeting the IntS11 ORF (Fig. 3B, lanes 5, 8), which depletes both endogenous and exogenously expressed IntS11 (Fig. 3A). This requirement for IntS11 endonuclease activity was also observed with the eGFP reporter driven by the MtnA promoter (Supplemental Fig. S4). Given that the 
Tatomer et al.

A

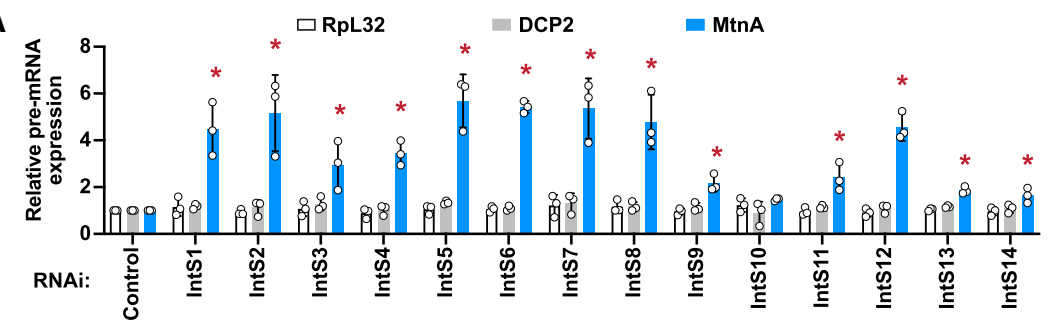

B

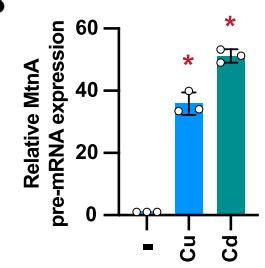

C

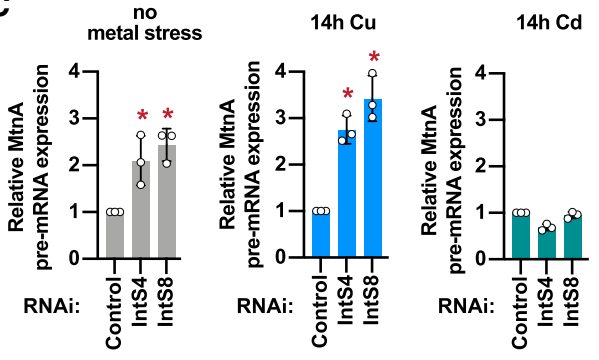

D

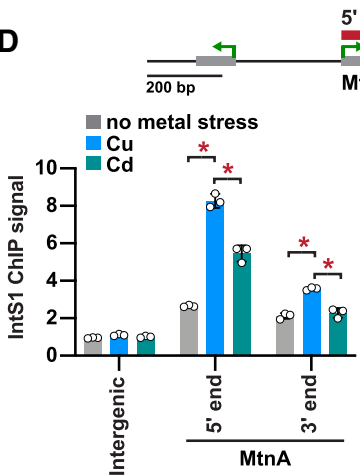

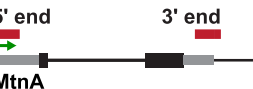

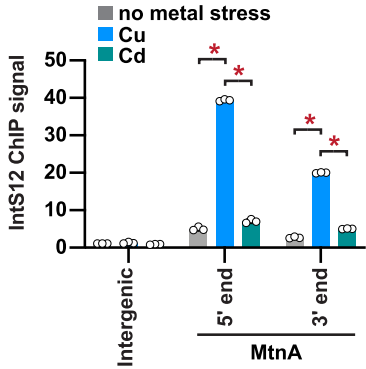

Figure 2. The Integrator complex is present at the MtnA locus during copper stress and attenuates MtnA transcription. (A) DL1 cells

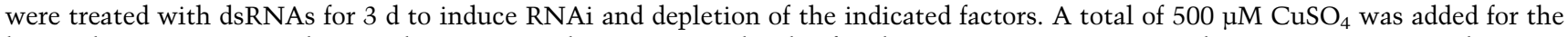
last $14 \mathrm{~h}$. RT-qPCR was then used to measure the pre-mRNA levels of endogenous RpL32, DCP2, and MtnA. Primer pairs that span an intron-exon boundary were used to specifically amplify pre-mRNAs. Data were normalized to RpL32 mRNA expression and are shown as mean $\pm \mathrm{SD}, N=3 .\left(^{*}\right) P<0.05$. (B) DL1 cells were unstressed $(-)$ or treated with $500 \mu \mathrm{M} \mathrm{CuSO}_{4}$ or $50 \mu M \mathrm{CdCl}_{2}$ for $14 \mathrm{~h}$, and RT-qPCR was then used to measure MtnA pre-mRNA levels. Data were normalized to RpL32 mRNA expression and are shown as mean $\pm \mathrm{SD}, N=3$. $\left({ }^{*}\right) P$ $<0.05$. (C) DL1 cells were treated with dsRNAs for $3 \mathrm{~d}$ and $500 \mu \mathrm{M} \mathrm{CuSO}_{4}$ or $50 \mu \mathrm{M} \mathrm{CdCl}_{2}$ was added for the final $14 \mathrm{~h}$, as indicated. RTqPCR was then used to measure MtnA pre-mRNA levels. Data were normalized to RpL32 mRNA expression and are shown as mean \pm SD, $N=3$. $\left({ }^{*}\right) P<0.05$. $(D)$ The MtnA locus with the locations of ChIP amplicons. Recruitment of IntS1 and IntS12 in unstressed cells (gray) or after the cells had been treated with $\mathrm{CuSO}_{4}$ (blue) or $\mathrm{CdCl}_{2}$ (green) for $14 \mathrm{~h}$ was measured using ChIP-qPCR. Data are shown as fold change relative to the IgG control (mean $\pm \mathrm{SD}, N=3) .\left({ }^{*}\right) P<0.05$.

E203Q mutation does not disrupt Integrator complex integrity (Baillat et al. 2005), these collective results indicate that the IntS11 endonuclease activity is required for Integrator to negatively regulate the transcriptional output of the MtnA promoter.

\section{The Integrator complex cleaves nascent MtnA mRNAs to trigger transcription termination}

By immunoprecipitating Integrator subunits followed by immunoblotting (Fig. 3C) or mass spectrometry (Fig. $3 \mathrm{D})$, we found that Integrator interacts with the nuclear RNA exosome, which catalyzes $3^{\prime}-5^{\prime}$ degradation of many RNAs (for review, see Zinder and Lima 2017). Interestingly, our genome-scale RNAi screen identified many RNA exosome core components and cofactors (including Rrp40 and Mtr4) as positive regulators of the MtnA promoter during copper stress (Fig. 1C,D). This suggests an interplay between the RNA exosome and Integrator complex and that the RNA exosome may also function in controlling MtnA transcriptional output. We confirmed that RNA exosome core components and cofactors were efficiently depleted by RNAi (Supplemental Fig. S5A), and that this resulted in the expected ribosomal RNA processing defects (Supplemental Fig. S5B) as well as decreased output from the MtnA promoter during copper stress (Fig. 3E; Supplemental Fig. S5C,D). We thus hypothesized that Integrator may cleave nascent MtnA transcripts to prematurely terminate RNAPII transcription (as Integrator is enriched near the $5^{\prime}$ end of the MtnA genomic locus during copper stress) (Fig. 2D) and that these cleaved transcripts are targeted for rapid degradation by the RNA exosome (Fig. 3F).

To test this model, we first treated cells with dsRNAs to deplete the exosome-associated RNA helicase Mtr4 (Lubas et al. 2011). This resulted in a reduction in fulllength endogenous MtnA mRNA expression (Fig. 3E; Supplemental Fig. S5E) and, concomitantly, a number of small RNAs were detected from the MtnA locus, including prominent transcripts with lengths of $\sim 85$ and $\sim 110$ nt (RNAs marked in orange) (Fig. 4A, lane 4). These small RNAs were dependent on the MTF- 1 transcription factor (Fig. 4A, lane 6) and capped at their $5^{\prime}$ ends, as they could be degraded by a $5^{\prime}$ phosphate-dependent exonuclease only after treatment with Cap-Clip Acid Pyrophophatase, which hydrolyzes cap structures to generate $5^{\prime}$ monophosphate groups (Supplemental Fig. S6A). Northern blots using multiple probes further indicated that the small RNAs have the same TSS as MtnA mRNA (Supplemental Fig. S6B) and ligation-mediated $3^{\prime}$ RACE revealed that these 
A
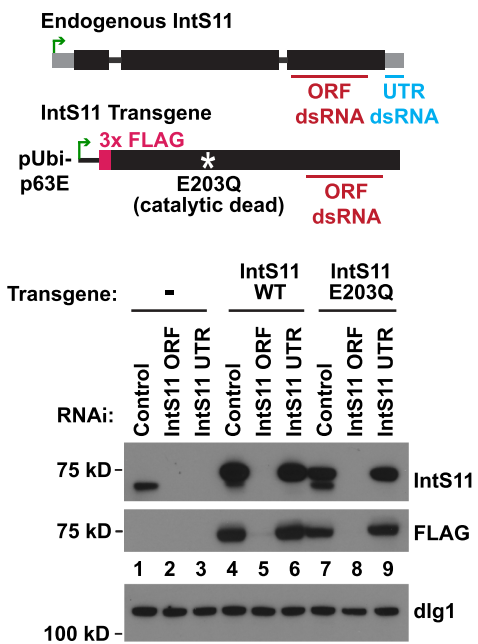

B
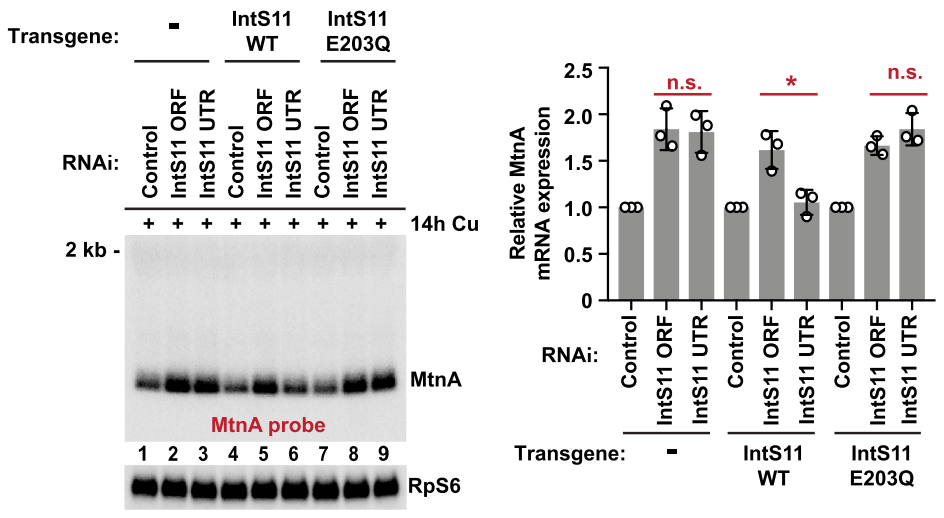

E

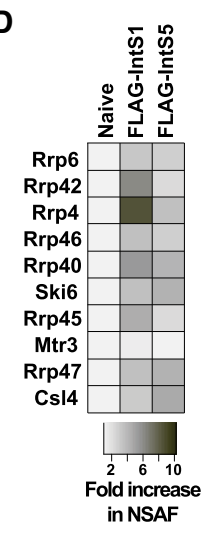

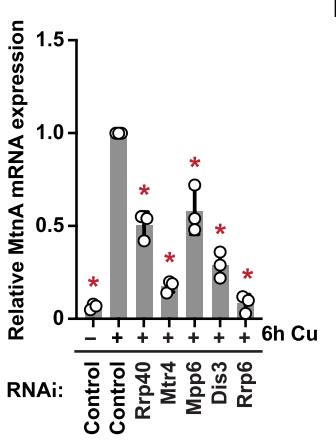

$\mathbf{F}$

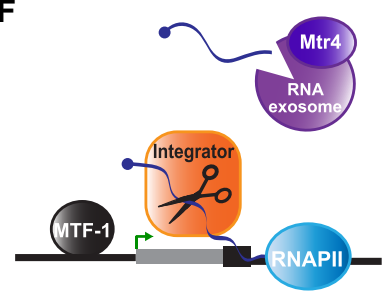

Figure 3. The IntS11 endonuclease activity and the RNA exosome regulate MtnA transcript levels. (A, top) Schematic of IntS11 knockdown/plasmid rescue strategy. The ORF dsRNA (red) depletes IntS11 generated from both the endogenous locus and transgenes that are driven by the ubiquitin-63E promoter (pUbi-p63E), while the UTR dsRNA (blue) only depletes endogenous IntS11. (Bottom) DL1 cells (lanes 1-3) or DL1 cells stably maintaining Flag-tagged wild-type (WT; lanes 4-6) or catalytically dead (E203Q; lanes 7-9) IntS11 transgenes were treated with the indicated dsRNAs for $3 \mathrm{~d}$. Western blot analysis was then used to examine IntS11 protein levels. dlg1 was used as a loading control. Representative blots are shown. $(B)$ Representative northern blot of the endogenous MtnA mRNA in DL1 cells stably maintaining IntS1 1 transgenes that had been treated with the indicated dsRNAs and $\mathrm{CuSO}_{4}$. Expression was quantified using ImageQuant and data are shown as mean $\pm \mathrm{SD}, N=3 .\left(^{*}\right) P<0.05$. $(C)$ Western blot analysis of $\mathrm{S} 2$ nuclear extracts that were subjected to purification using Flag affinity resin. (Left panel) Western blots showing input levels of the denoted proteins in nuclear extracts derived from naïve S2 cells, S2 cells stably expressing Flag-IntS1, or S2 cells stably expressing Flag-IntS5. (Right panel) same as left panel except that Flag immunoprecipitates were probed. $(D)$ Heat map showing the relative enrichment of RNA exosome components within Flag-Integrator subunit purifications as determined by mass spectrometry. Normalized spectral abundance factor (NSAF) values were quantified as previously described (Zybailov et al. 2006), and fold-enrichment of each RNA exosome subunit in Integrator purifications relative to purification from naïve extract is given. (E) DL1 cells were treated with dsRNAs for $3 \mathrm{~d}$ to induce RNAi and depletion of the indicated factors. $\mathrm{CuSO}_{4}$ was added for the last $6 \mathrm{~h}$. Northern blots were then used to quantify expression of endogenous MtnA mRNA and data are shown as mean $\pm \mathrm{SD}, N=3$. $\left({ }^{*}\right) P<0.05$. $(F)$ Model for Integrator-dependent premature termination. After cleavage of the nascent MtnA transcript by IntS11, the small RNAs are degraded by the RNA exosome.

small RNAs had detectable 3' oligoadenylation, a mark known to facilitate RNA degradation by the RNA exosome (Supplemental Fig. S6C,D; Supplemental Table S2; LaCava et al. 2005)

We next codepleted Mtr4 and Integrator subunits and observed that these small RNAs were completely eliminated and that full-length MtnA mRNA expression was restored (Fig. 4A, lanes 8,10,12; Supplemental Fig. S5E). This suggests that generation of the small RNAs is dependent on Integrator. In support of this, in cells in which the endogenous IntS11 protein had been depleted by RNAi (Fig. 4B, lane 4), reexpression of a wild-type (WT) IntS11 transgene restored expression of the MtnA small RNAs (Fig. 4B, lane 8), whereas expression of a catalytically dead (E203Q) (Baillat et al. 2005) IntS11 transgene did not (Fig. 4B, lane 12; Supplemental Fig. S7).

Together, the data in Figures 1-4 support a model in which the Integrator complex is recruited to the active MtnA locus during copper stress to cleave nascent RNAs, thereby facilitating premature termination (Fig. 3F). 
A

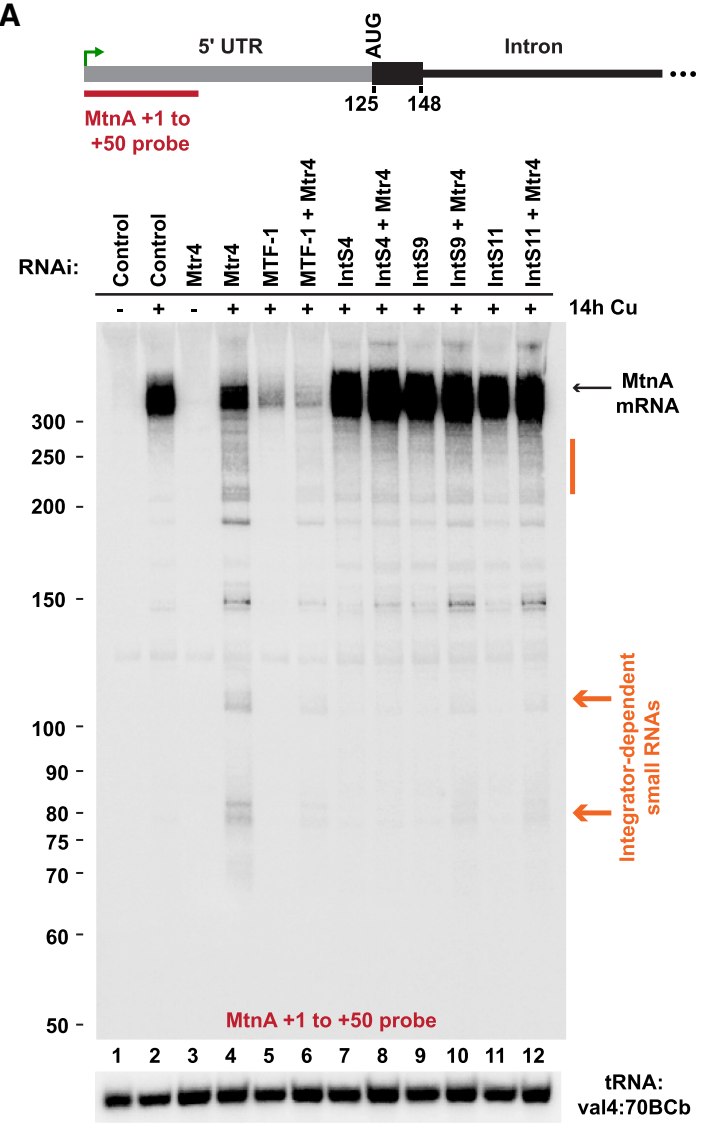

B

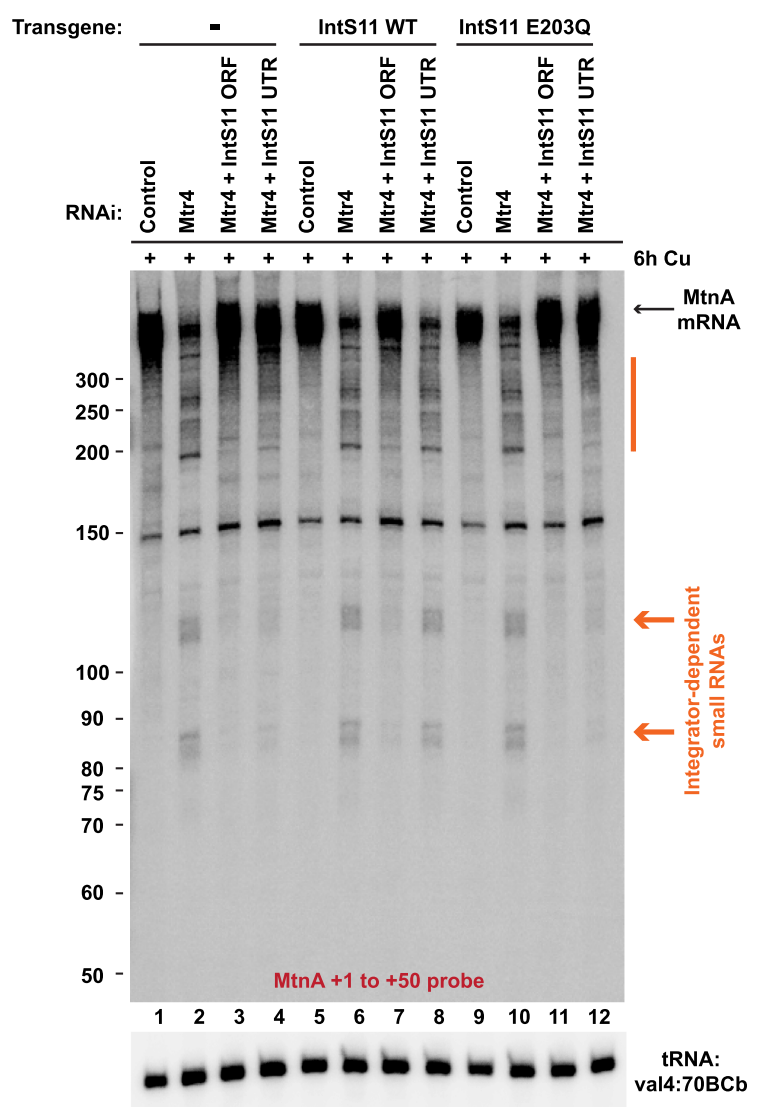

Figure 4. The Integrator complex cleaves nascent MtnA RNAs to catalyze premature transcription termination. $(A)$ Northern blotting was used to analyze RNAs generated from the endogenous MtnA locus in DL1 cells treated with the indicated dsRNAs and CuSO 4 . Fulllength MtnA mRNA (black arrow) and Integrator-dependent small RNAs (orange) are indicated. (B) Parental DL1 cells (lanes 1-4) or DL1 cells stably expressing WT (lanes 5-8) or catalytically inactive (lanes 9-12) IntS11 transgenes were treated with the indicated dsRNAs and $\mathrm{CuSO}_{4}$. Northern blotting was then performed as in $A$.

This role for Integrator at MtnA is mechanistically related to its function at snRNA genes, where Integrator both cleaves the nascent snRNA and promotes RNAPII termination/recycling. Rather than Integrator attaining a novel function at protein-coding genes (Gardini et al. 2014; Stadelmayer et al. 2014; Skaar et al. 2015), our data show that the Integrator endonuclease activity has been "repurposed" at the MtnA gene. Once generated, the prematurely terminated small RNAs are actively targeted for $3^{\prime}-5^{\prime}$ degradation by the nuclear RNA exosome, at least in part due to 3' oligoadenylation. Degradation of these small RNAs appears to be critical for enabling subsequent rounds of MtnA transcription, as the output of the MtnA promoter is reduced when the RNA exosome is depleted from cells (Figs. 1C, 3E). The requirement of the RNA exosome for MtnA transcription is, however, abrogated when Intergator is depleted or catalytically inactive. These results indicate a potential epistastic relationship in which Integrator cleaves nascent RNAs that must be subsequently degraded to allow production of more full-length mRNA transcripts in copper stressed cells.
Many Drosophila protein-coding genes are controlled by the Integrator complex

As Integrator-dependent termination events potently attenuate transcription from the MtnA promoter during copper stress, we next asked whether additional proteincoding genes are similarly regulated. IntS9, which forms a heterodimer with IntS11 and is essential for its endonuclease activity (Wu et al. 2017), was depleted from DL1 cells for $3 \mathrm{~d}$ and copper was added for the final $14 \mathrm{~h}$. Using RNA-seq, we identified 409 and 49 genes that were upand down-regulated, respectively, upon IntS9 depletion (fold change $>1.5$ and $P<0.001$ ) (Fig. 5A; Supplemental Table S3). The set of up-regulated mRNAs was enriched in genes that respond to stimuli as well as gene ontology categories related to cell migration, proliferation, and cellfate specification (Supplemental Fig. S8). In contrast, no gene ontology categories were enriched in the set of down-regulated mRNAs.

To validate the RNA-seq results, seven mRNAs that had differing magnitudes of fold change upon IntS9 depletion were selected for further analysis (genes marked in 
A

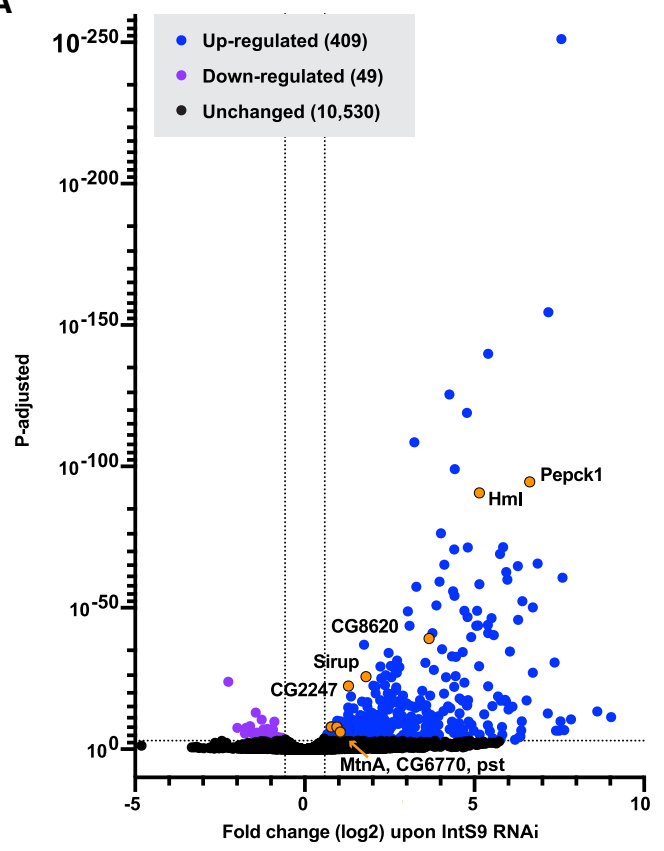

B

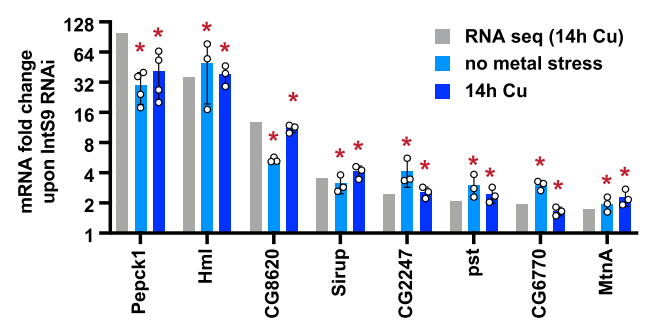

C

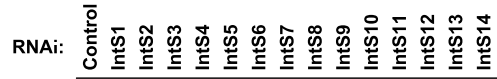
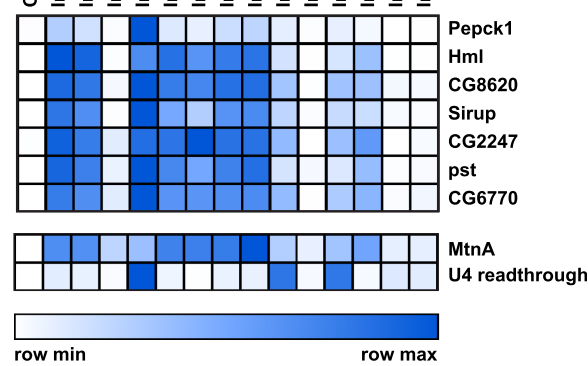

D

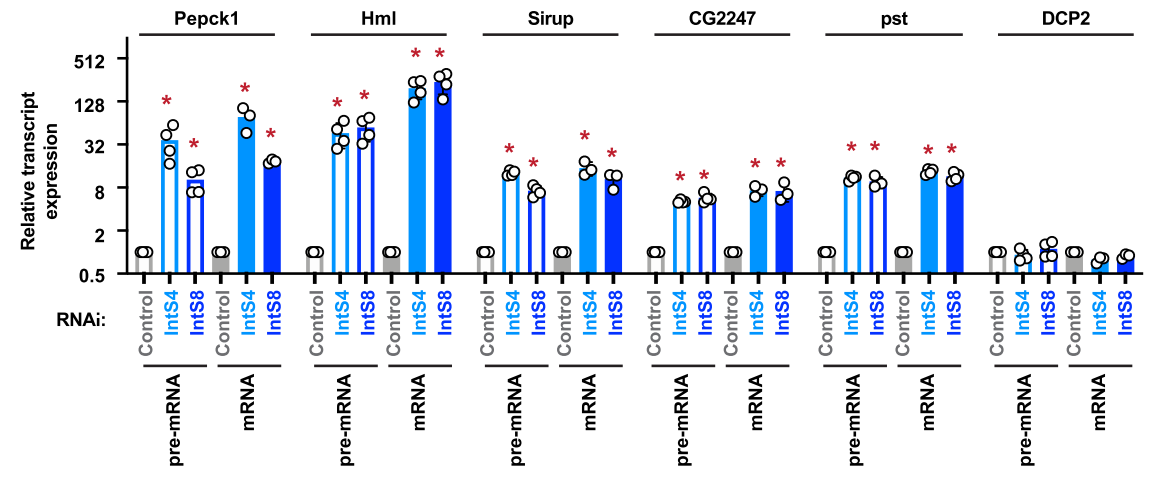

$\mathbf{E}$

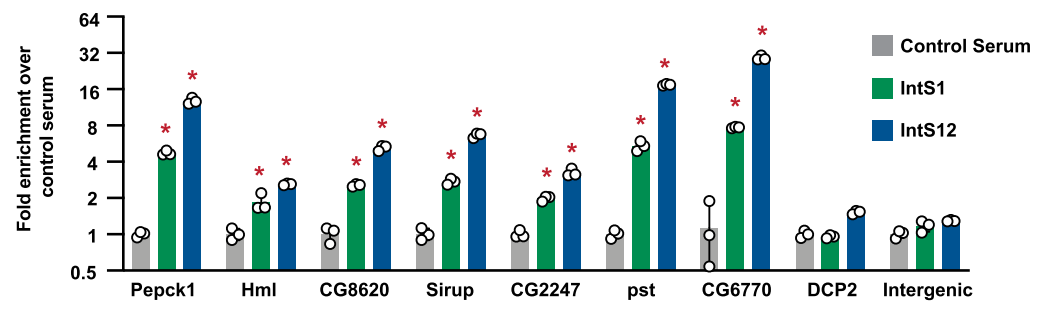

Figure 5. Integrator depletion results in up-regulation of many protein-coding genes. $(A)$ DL1 cells were treated for $3 \mathrm{~d}$ with a control ( $\beta$ gal) dsRNA or a dsRNA to deplete IntS9, and $\mathrm{CuSO}_{4}$ was added for the last $14 \mathrm{~h}$. Total RNA was isolated, depleted of ribosomal RNAs, and RNA-seq libraries prepared (three biological replicates per condition). The magnitude of change in mRNA expression compared with statistical significance $(P$-value) is shown as a volcano plot. Threshold used to define IntS9-affected mRNAs was fold change $>1.5$ and $P<0.001$. (B) To verify the RNA-seq results (gray), DL1 cells were treated for $3 \mathrm{~d}$ with a control ( $\beta g a l)$ dsRNA or a dsRNA to deplete IntS9 with or without $\mathrm{CuSO}_{4}$ added for the last $14 \mathrm{~h}$ (light blue and dark blue, respectively). RT-qPCR was then used to quantify changes in mRNA expression levels. Data were normalized to RpL32 mRNA expression and are shown as mean \pm SD compared with treatment with a

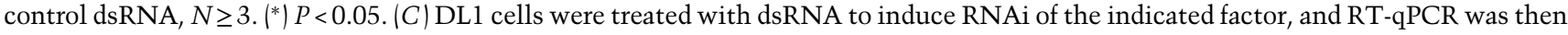
used to quantify changes in mRNA expression levels. $\mathrm{CuSO}_{4}$ was added for the last $14 \mathrm{~h}$ only when measuring MtnA mRNA levels. Northern blotting was used to quantify readthrough transcription downstream from the U4:39B snRNA as described in Figure 1E. Data are summarized as a heat map using Morpheus (Broad Institute) with darker shades representing increased transcript expression compared with treatment with a control ( $\beta$ gal) dsRNA. Individual RT-qPCR data points are provided in Supplemental Figure S9. (D) RT-qPCR was used to measure the mRNA and pre-mRNA levels of the indicated transcripts. Data were normalized to RpL32 mRNA expression and are shown as mean $\pm \mathrm{SD}, N \geq 3$. $\left(^{*}\right) P<0.05$. $(E)$ ChIP-qPCR was used to measure IntS1 and IntS12 occupancy at the indicated promoter regions. Data are shown as fold change relative to the IgG control serum (mean $\pm \mathrm{SD}, N=3$ ). $\left({ }^{*}\right) P<0.05$. 
orange in Fig. 5A). Among these genes, five contain introns and two are intronless (CG8620 and CG6770). RT-qPCR confirmed that expression of all seven of these mRNAs increased upon IntS9 depletion regardless of whether the cells were subjected to metal stress (Fig. $5 B)$. Therefore, we did not induce metal stress in subsequent experiments. Upon depleting each Integrator subunit individually, we noted that expression of these mRNAs was often most affected by depletion of IntS4, which forms the scaffold of the Integrator cleavage module (Fig. 5C; Supplemental Fig. S9; Albrecht et al. 2018). Moreover, analogous to our prior results with MtnA (Fig. 1E), depletion of many non-catalytic Integrator subunits (notably IntS1, IntS2, IntS5, IntS6, IntS7, and IntS8) also caused large increases in the expression of these mRNAs (Fig. 5C; Supplemental Fig. S9).

\section{The Integrator complex cleaves many nascent mRNAs} to trigger transcription termination

To determine whether Integrator controls the outputs of these protein-coding genes transcriptionally or post-transcriptionally, we measured pre-mRNA levels from the intron-containing genes. Expression of these pre-mRNAs increased upon depletion of Integrator subunits, and the observed fold changes are similar to the increases in mature mRNA levels (Fig. 5D). These results mirror our findings at MtnA (Fig. 2A) and strongly suggest that the observed increases in mature transcript levels are due to transcriptional control by Integrator, and not due to indirect effects of Integrator functioning in snRNA processing (Supplemental Fig. S3A,B). Moreover, ChIP-qPCR confirmed that multiple Integrator subunits are recruited to the $5^{\prime}$ ends of these gene loci (Fig. 5E). As an additional control, we monitored the DCP2 locus and observed minimal Integrator binding to the gene (Fig. 5E) as well as no change in DCP2 pre-mRNA or mRNA levels upon Integrator depletion (Fig. 5D).

To further confirm that Integrator regulation of these genes was driven by their promoters, we cloned each of these regions (along with a portion of the $5^{\prime}$ UTRs) upstream of an eGFP reporter (Fig. 6A). Indeed, the expression of eGFP mRNA driven from each of the examined promoters was sensitive to the levels of Integrator subunits, including those in the Integrator cleavage module (especially IntS4) as well as many of the non-catalytic subunits (Fig. 6A; Supplemental Fig. S10). Similar results were obtained with the MtnA-driven eGFP reporter (Fig. 6A), whereas a reporter plasmid that monitors Integrator activity downstream from an snRNA (Chen et al. 2013) displayed a distinct sensitivity pattern (Fig. 6B). For example, IntS6 depletion caused up-regulation of the output from all the Integrator regulated protein-coding gene promoters (Fig. 6A), but had minimal effect on the U4:39B snRNA readthrough reporter (Fig. 6B; Supplemental Fig. S10). As an additional control, we confirmed that expression of an eGFP reporter driven by the ubiquitin-63E (Ubi-p63e) promoter did not increase upon depletion of any of the Integrator subunits (Fig. 6A; Supplemental Fig. S10). This is consistent with the RNA-seq results

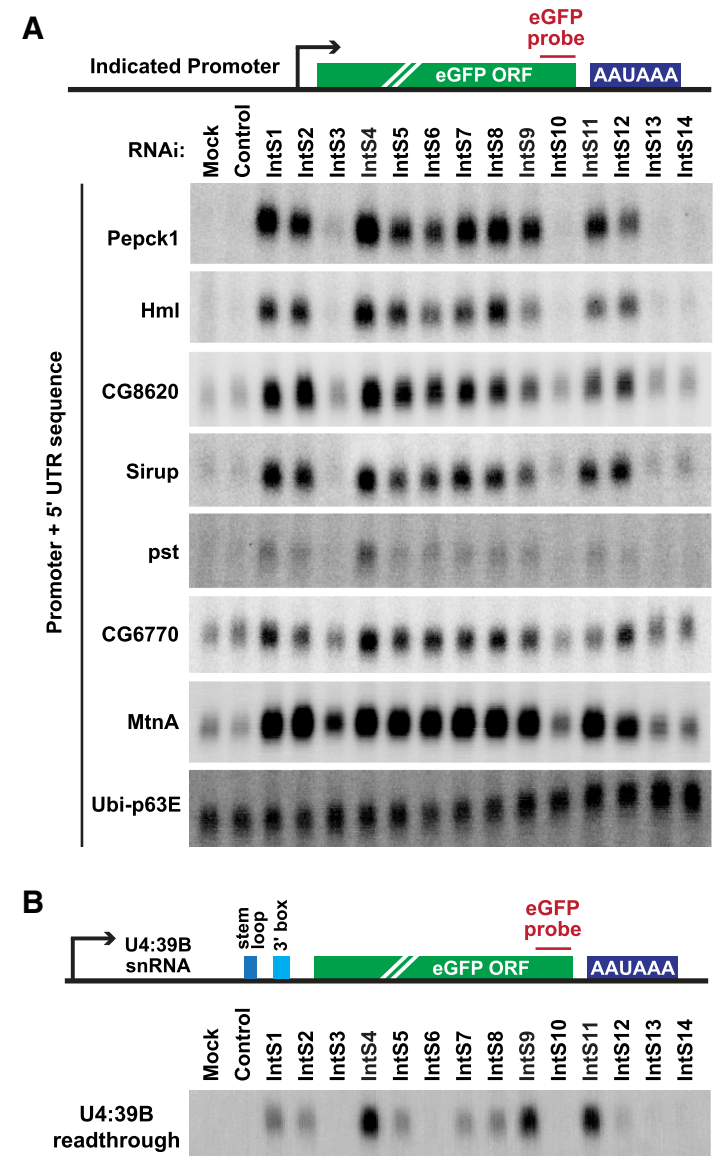

Figure 6. eGFP reporter genes driven by the exemplar promoters are regulated by Integrator. $(A)$ The promoter and $5^{\prime}$ UTR of each of the indicated protein-coding genes was cloned upstream of an eGFP reporter. The plasmids were then individually transfected into DL1 cells that had been treated with the indicated dsRNAs. $\mathrm{CuSO}_{4}$ was added for the last $14 \mathrm{~h}$ only when measuring eGFP production from the MtnA promoter. Northern blots were used to quantify expression of each eGFP reporter mRNA. Representative blots are shown. Loading controls and quantification are provided in Supplemental Figure S10. (B) DL1 cells were treated with the indicated dsRNAs and then transfected with a reporter plasmid that produces eGFP when the encoded U4:39B snRNA fails to be properly processed at its $3^{\prime}$ end. Northern blots were used to quantify eGFP mRNA expression that is a result of U4:39B readthrough. A representative blot is shown. Loading controls and quantification are provided in Supplemental Figure S10.

that showed endogenous Ubi-p63e mRNA levels do not change upon Integrator depletion (Supplemental Table S3).

Next, we tested whether Integrator catalyzes premature transcription termination at these genes in a manner analogous to how it controls MtnA. We first investigated whether the IntS11 endonuclease activity is required. Depletion of the endogenous IntS11 protein using a dsRNA targeting the IntS11 3' UTR (Fig. 3A) resulted in increased expression of each of the examined Integratordependent mRNAs (Fig. 7A). Expression of a wild-type (WT) IntS11 transgene restored mRNA expression to 
A
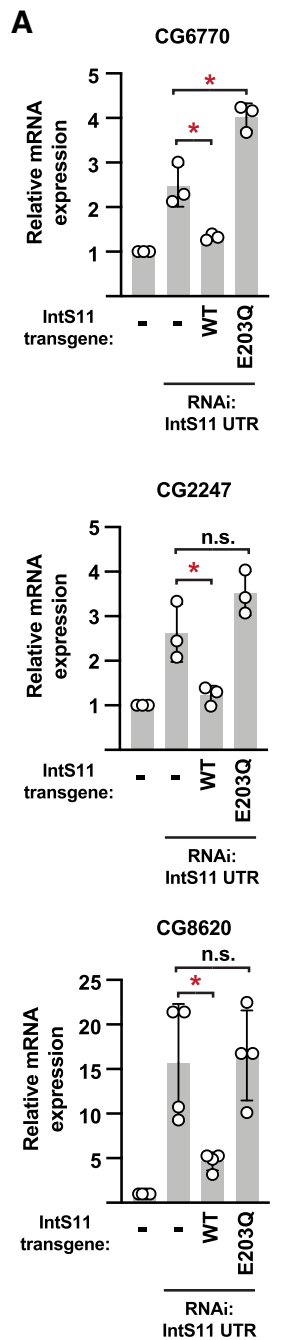
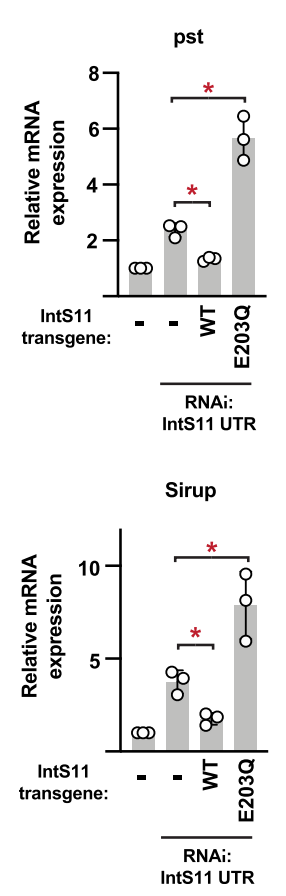

C
B

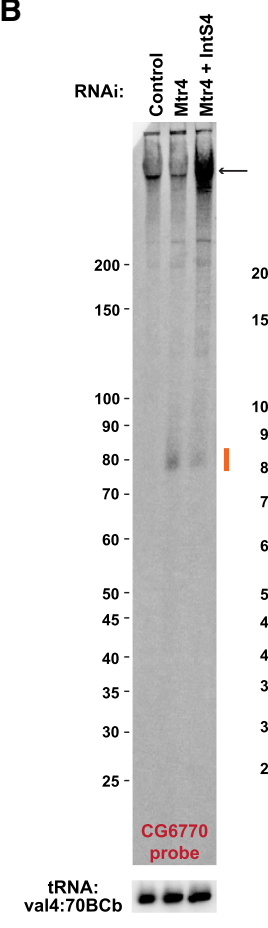

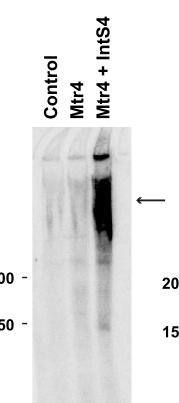

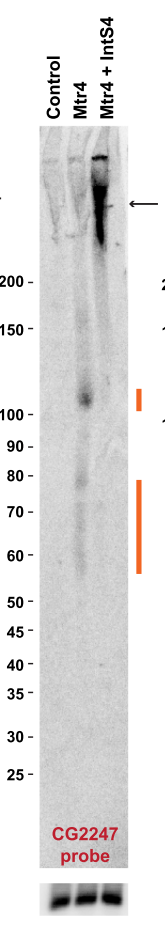

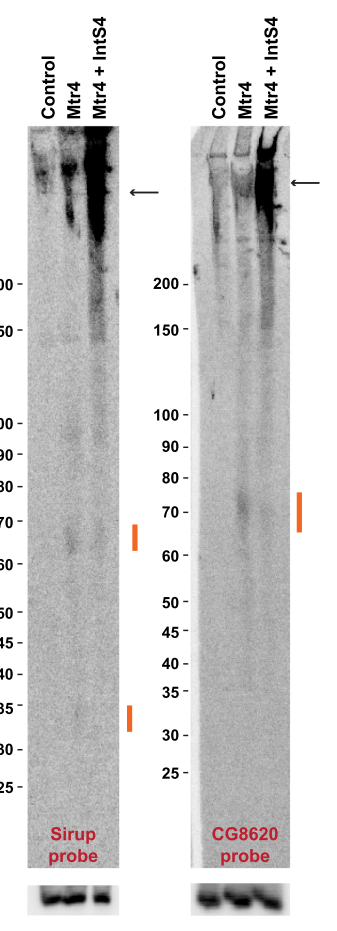

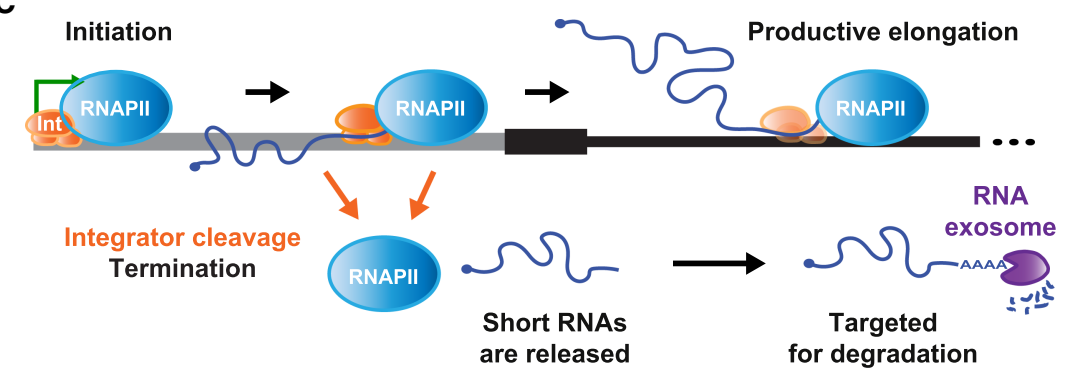

Productive elongation

for degradation

Figure 7. The Integrator complex cleaves many nascent mRNAs to catalyze premature transcription termination. (A) Parental DL1 cells (denoted-) or DL1 cells stably expressing WT or catalytically inactive (E203Q) IntS11 transgenes were treated with a dsRNA to the IntS11 $3^{\prime}$ UTR, thereby depleting endogenous IntS11 but not IntS11 made from the transgenes. RT-qPCR was then used to quantify expression of the indicated mRNAs. Data were normalized to RpL32 mRNA expression and are shown as mean $\pm \mathrm{SD}, N=3$. (*) $P<0.05$. $(B)$ DL1 cells were treated with dsRNAs for $3 \mathrm{~d}$ to induce RNAi and depletion of the indicated factors. Northern blotting using $50 \mu \mathrm{g}$ of total RNA was then used to analyze transcripts from the $5^{\prime}$ ends of the indicated protein-coding loci. Integrator-dependent small RNAs (orange) and full-length mRNAs (black arrows) are noted. Representative blots are shown. $(C)$ Schematic of a protein-coding locus, highlighting the presence of Integrator (Int, orange) and possible fates of RNAPII (blue). After transcription initiation, RNAPII can transition into productive elongation to generate the mature mRNA. Alternatively, Integrator can cleave the nascent RNA, thereby enabling transcription termination and degradation of the short RNA by the nuclear RNA exosome (purple).

levels similar to control treated cells, whereas the catalytically dead IntS11 E203Q mutant did not (Fig. 7A). The IntS11 endonuclease activity is thus indeed required for regulation of each of these genes and, notably, the presence of the E203Q mutant protein exacerbated the changes in expression of the CG6770, pst, and Sirup mRNAs, potentially indicative of a dominant negative effect.

Northern blots were then used to detect premature termination products from each of the Integrator-regulated genes (Fig. 7B). Given that the MtnA cleavage products are rapidly degraded by the RNA exosome (Fig. 4), we reasoned that small RNAs generated from other loci would likewise be unstable. Depletion of the exosome-associated RNA helicase Mtr4 (Lubas et al. 2011) enabled small RNAs to be detected from the $5^{\prime}$ ends of the Integratordependent genes, and these transcripts were lost upon Integrator codepletion (RNAs marked in orange; Fig. 7B). Interestingly, these small RNAs were of defined lengths and often 50-110 nt, roughly mirroring the sizes of cleavage products observed at the MtnA locus (Fig. 4). We thus conclude that the Integrator complex is recruited to a number of protein-coding genes where it cleaves nascent RNAs and facilitates premature transcription termination (Fig. 7C). 
Integrator cleavage of nascent mRNAs is likely independent of a $3^{\prime}$ box sequence

At snRNA gene loci, a conserved but relatively degenerate sequence known as the $3^{\prime}$ box is located 9-19 nt downstream from the $3^{\prime}$ ends of mature snRNA transcripts and is required for Integrator cleavage (Hernandez 1985; Baillat and Wagner 2015). A similar 3' box-like sequence is not immediately recognizable within MtnA or the other transcripts we studied in detail, and we thus introduced deletions into the MtnA 5' UTR upstream of the eGFP reporter in an attempt to alter the cleavage product sizes (Supplemental Fig. S11A). Notably, this analysis revealed that Integrator-dependent small RNAs derived from the MtnA promoter appear to be largely 70-90 nt in length, regardless of the mRNA sequence (Supplemental Fig. S11B). This suggests that Integrator may cleave nascent mRNAs at a set distance from the TSS in a manner independent of local DNA or RNA sequence content, perhaps at positions of RNAPII pausing/stalling or nucleosomes /Chiu et al. 2018).

\section{Discussion}

Altogether, our data indicate that the Integrator complex can attenuate the expression of protein-coding genes by catalyzing premature transcription termination (Fig. 7C). The IntS11 endonuclease cleaves a subset of nascent mRNAs, which ultimately triggers degradation of the transcripts by the RNA exosome along with RNAPII termination. We suggest that many protein-coding genes are negatively regulated via this attenuation mechanism, and the Drosophila MtnA promoter highlights contextspecific regulation by Intgerator. Transcription of MtnA is induced by copper or cadmium stress, and yet we find that Integrator is robustly recruited to the MtnA promoter only under copper stress conditions (Fig. 2). This is not because the Integrator complex is generally diassembled or "poisoned" by cadmium, as Integrator continues to regulate the outputs of other protein-coding genes (Supplemental Fig. S12). We instead propose that contextspecific regulation of this locus may be related to the fact that cadmium is a strictly toxic metal, while copper is required for the function of a subset of enzymes and must be maintained in a narrow concentration range (for review, see Festa and Thiele 2011). Therefore, homeostatic control of MtnA is required to maintain copper levels, while cells need to maximally produce MtnA in the presence of cadmium. We thus propose that regulation of MtnA levels by Integrator during copper stress is for fine-tuning purposes, perhaps to limit maximal transcriptional induction and/or facilitate transcriptional shut-off once copper stress has passed. Our results suggest that the Integrator complex can be recruited to gene loci only when needed, thereby ensuring tight control over transcriptional output.

In addition to cleaving MtnA transcripts, Integrator cleaves multiple other RNA classes in metazoan cells, including enhancer RNAs (Lai et al. 2015), snRNAs (Baillat et al. 2005), telomerase RNA (Rubtsova et al. 2019), and some herpesvirus microRNA precursors (Cazalla et al. 2011; Xie et al. 2015). Using RNA-seq, we expanded this list of Integrator target loci and identified hundreds of additional protein-coding genes that are negatively regulated by Integrator (Fig. 5). We focused on a set of Integratordependent genes and found that Integrator catalyzes premature transcription termination of these genes (Figs. 6, 7), which is consistent with prior studies that suggested roles for Integrator in termination (Skaar et al. 2015; Shah et al. 2018; Gómez-Orte et al. 2019). Some of these genes (CG8620, Pepck1, and Sirup) have promoter-proximal RNAPII that rapidly turns over (Shao and Zeitlinger 2017), which may indicate that Integrator can aid in clearing paused or stalled RNAPII. Once Integrator has cleaved the nascent mRNAs, we find that they are rapidly degraded from their $3^{\prime}$ ends by the RNA exosome (Fig. 7C). This may be critical for enabling subsequent rounds of transcription (especially at the MtnA locus), perhaps because the small RNAs can form stable RNA-DNA hybrids (R-loops) that block transcription initiation or elongation (Belotserkovskii et al. 2017; Nojima et al. 2018; Crossley et al. 2019).

Endonucleolytic cleavage is critical for Integrator regulation at snRNA and protein-coding genes, but our data indicate that these loci have different dependencies on Integrator subunits. Our genetic studies indicate that Integrator subunits 4, 9, and 11 (which form the Integrator cleavage module) are most important for snRNA processing, while the non-catalytic Integrator subunits (all of which currently lack annotated molecular functions) play minor roles (Figs. 1E, 6B). In contrast, large increases in mRNA expression were observed when many of the non-catalalytic subunits were depleted (especially IntS1, IntS2, IntS5, IntS6, IntS7, and IntS8) (Figs. 1E, 5C, 6A). IntS13 was recently shown to be able to function independently from other Integrator subunits at enhancers (Barbieri et al. 2018), suggesting the existence of submodules or "specialized" complexes that may enable the activity and function of Integrator to be distinctly regulated depending on the gene locus and cellular state. Future work will reveal the subunit requirements of Integrator complexes at distinct loci and clarify the interplay between IntS11 endonuclease activity and other Integrator subunits. For example, the non-catalytic subunits may be critical for the formation and targeting of the complex to specific loci and/or controlling RNAPII dynamics.

Finally, we note that the metazoan Integrator complex has parallels with the yeast Nrd1-Nab3-Sen1 (NNS) complex that (1) terminates transcription at both mRNA and snRNA loci and (2) interacts with the RNA exosome (for review, see Arndt and Reines 2015). Interestingly, the underlying molecular mechanisms of transcription termination carried out by these two complexes are quite distinct. NNS uses the Sen 1 helicase to pull the nascent transcript out of the RNAPII active site, while Integrator likely promotes termination by taking advantage of its RNA endonuclease activity and providing an entry site for a $5^{\prime}-3^{\prime}$ exonuclease (Connelly and Manley 1988; Eaton et al. 2018). There is currently conflicting data on whether the canonical "torpedo" exonuclease Rat1/Xrn2 is involved 
in termination at snRNA genes as only subtle termination defects have been observed at these loci when Rat1/Xrn2 is depleted from cells (O'Reilly et al. 2014; Fong et al. 2015; Eaton et al. 2018). Notably, Cpsf73 has been shown to behave as both an endonuclease and exonuclease (Yang et al. 2009), raising the possibility that IntS11 could support a "Rat1/Xrn2-like" function and mediate termination. Future studies that compare and contrast the Integrator and NNS complexes, especially how their recruitment and termination activities are controlled, will shed light on this important facet of gene regulation. In summary, transcription attenuation through premature termination was first described decades ago in bacteria (for review, see Naville and Gautheret 2010), and our work indicates that the metazoan Integrator complex can function analogously to limit expression from protein-coding genes.

\section{Materials and methods}

Drosophila cell culture and plasmid transfections

Drosophila DL1 cells were cultured at $25^{\circ} \mathrm{C}$ in Schneider's Drosophila medium (Thermo Fisher Scientific 21720024), supplemented with $10 \% \quad(\mathrm{v} / \mathrm{v})$ fetal bovine serum (HyClone SH30910.03), 1\% (v/v) penicillin-streptomycin (Thermo Fisher Scientific 15140122), and 1\% (v/v) L-glutamine (Thermo Fisher Scientific 35050061). Drosophila S2 cells were cultured at $25^{\circ} \mathrm{C}$ in SFX-Insect (HyClone SH30278.01).

To generate eGFP and nLuc reporter plasmids, the indicated sequences were inserted into pMtnA eGFP SV40 (Kramer et al. 2015). To generate Integrator expression plasmids, each Drosophila cDNA was cloned into a previously described pUB-3xFlag vector (Chen et al. 2012). Cloning details for all constructs and how they were transfected using Effectene (Qiagen 301427) or Fugene HD (Promega E2311) are provided in the Supplemental Material.

\section{Genome-scale RNAi screen}

Double-stranded RNAs (250 ng/well) were pre-spotted in 384well plates (Ambion AM8500). DL1 cells stably maintaining "pMtnA eGFP MALAT1" were grown for one passage in the absence of hygromycin B. A total of 15,000 cells were then seeded in each well of the 384-well plates in $10 \mu \mathrm{L}$ of serum-free Schneider's Drosophila media. After $1 \mathrm{~h}$, complete media (with serum) was added $(20 \mu \mathrm{L} /$ well). Cells were grown for $3 \mathrm{~d}$ and then treated with $10 \mu \mathrm{L}$ of media containing $\mathrm{CuSO}_{4}(500 \mu \mathrm{M}$ final concentration, Fisher BioReagents BP346-500) for 6 h. Cells were fixed (5\% formaldehyde, Fisher BioReagents BP531-500) and stained with Hoechst 33342 (Sigma B2261) to visualize nuclei. Four images per well (eGFP and Hoechst 33342 staining) were captured at 20x magnification using an automated microscope (ImageXpress Micro, Molecular Devices) and analyzed using MetaXpress software. "Mean stain integrated intensity" of eGFP and "total cell number" were calculated for each image, and the median and interquartile ranges (IQR) were used to calculate a $z$-score for each plate: (Mean stain integrated intensity-median)/ (IQR*74). Wells with robust $Z$-scores $\geq 1.3$ or $\leq-1.3$ were considered hits and Gene Ontology (GO) analysis was performed using Database for Annotation, Visualization, and Integrated Discovery (DAVID), v6.8 (https://david.ncifcrf.gov/home.jsp) with standard parameters.
RNAi

Double-stranded RNAs (dsRNAs) from the DRSC (Drosophila RNAi Screening Center) were generated by in vitro transcription (MEGAscript kit, Thermo Fisher Scientific AMB13345) of PCR templates containing the $\mathrm{T} 7$ promoter sequence on both ends. Primer sequences are provided in Supplemental Table S4. Complete details of how cells were seeded, transfected (if applicable), and processed are provided in the Supplemental Material.

Analysis of RNA expression

Total RNA was isolated using TRIzol (Thermo Fisher Scientific 15596018) as per the manufacturer's instructions. To detect full-length mature mRNAs, northern blots using NorthernMax reagents (Thermo Fisher Scientific) and oligonucleotide probes were performed as previously described (Tatomer et al. 2017). Small RNAs were separated by $8 \%$ denaturing polyacrylamide gel electrophoresis (National Diagnostics EC-833) and electroblotted/UV crosslinked to Hybond N+ membrane (GE Healthcare RPN303B). ULTRAhyb-oligo hybridization Buffer (Thermo Fisher Scientific AM8663) was used as per the manufacturer's instructions. All oligonucleotide probe sequences are provided in Supplemental Table S4. Blots were viewed and quantified with the Typhoon 9500 scanner (GE Healthcare) and quantified using ImageQuant (GE Healthcare).

For analysis of transcript levels by RT-qPCR, cDNA was reversed transcribed from total RNA using TaqMan Reverse Transcription Reagents (Thermo Fisher Scientific N8080234) according to the manufacturer's instructions. Random hexamers were used, except for Supplemental Figure S5A where oligo(dT) primer was used. RT-qPCR was then carried out in triplicate using Power SYBR Green Master Mix (Thermo Fisher Scientific 4367659). All RTqPCR primers are provided in Supplemental Table S4.

The $5^{\prime}$ cap status of the MtnA small RNAs was characterized using Cap-Clip Acid Pyrophosphatase (CellScript C-CC15011H) and Terminator 5'-phosphate-dependent exonuclease (Lucigen TER51020). Ligation-mediated RACE was used to determine the $3^{\prime}$ ends of the MtnA small RNAs and full details are provided in the Supplemental Material.

Analysis of protein expression

Protein levels were measured using standard western blotting or immunofluorescence protocols and the details are provided in the Supplemental Material. Antibodies used and their conditions are provided in Supplemental Table S5.

\section{Chromatin immunoprecipitation}

ChIP-qPCR was performed as described in the Supplemental Material. Antibodies used and their conditions are provided in Supplemental Table S5. Primers used are provided in Supplemental Table S4.

\section{IP/Mass spectrometry analysis}

Nuclear extracts were prepared from S2 cell lines stably expressing Flag-IntS1, Flag-IntS5, and naïve S2 cells as a negative control. Purifications were conducted using $\sim 10 \mathrm{mg}$ of nuclear extract $(2$ $\mathrm{mL})$. Details for Flag affinity purification, sample digestion, nanoLC MS/MS analysis and database searching are provide in the Supplemental Material. 
RNA-seq

DL1 cells were treated for $3 \mathrm{~d}$ with a control (Bgal) dsRNA or a dsRNA to deplete IntS9. $\mathrm{CuSO}_{4}$ was added for the last $14 \mathrm{~h}$. Total RNA was isolated from three biological replicates and 1 $\mu \mathrm{g}$ of each was treated with DNase I (Qiagen 79254) and purified (RNeasy MinElute Cleanup Kit, Qiagen 74204). RNA quality was confirmed with a TapeStation (Agilent). RNA-seq libraries were generated using the TruSeq Stranded Total RNA preparation kit (Illumina RS-122-2302) and sequenced using a single-end 75 bp cycle run on an Illumina NextSeq 500. Details for how reads were mapped and analyzed are provided in the Supplemental Material. All RNA-seq data sets generated in this study are available for download from GEO (GSE136150). Data can also be viewed on the UCSC browser using the following link: https://genome.ucsc .edu/s/meishengxiao/dm6_DL1_Bgal_IntS9_WiluszLab_Upenn.

Quantification and statistical analysis

For northern blots, RT-qPCRs, and automated microscopy experiments, statistical significance for comparisons of means was assessed by Student's $t$-test. Unless otherwise indicated, the comparison was to the control ( $\beta$ gal dsRNA) treated samples. Statistical details and error bars are defined in each figure legend.

\section{Acknowledgments}

We thank Todd Albrecht for his help in generating Drosophila Integrator antibodies, the Andrulis lab for the Rrp40 antibody, William K. Russel and the UTMB Proteomics Core, the University of Pennsylvania High-Throughput Screening Core, Dan Beiting and Ana Misic for help with RNA-seq library preparation, as well as Karen Adelman and other members of the Cherry, Wagner, and Wilusz labs for helpful discussions. This work was supported by National Institutes of Health grants R35-GM119735 (J.E.W.), K99-GM131028 (D.C.T.), R01-AI122749 (S.C.), R01-AI074951 (S.C.), and R01-GM134539 (E.J.W.), and Welch Foundation grant $\mathrm{H}-1889$ (E.J.W.). S.C. is a recipient of the Burroughs Wellcome Investigators in the Pathogenesis of Infectious Disease Award. J.E.W. is a Rita Allen Foundation Scholar.

Author contributions: D.C.T., S.C., and J.E.W. conceived the project and designed/analyzed the RNAi screen. D.C.T., N.D.E., D.L., J.Z.J., M.J., and K.-L.H. performed experiments and analyzed data. M.-S.X. analyzed the RNA-seq results. D.C.T., E.J.W., S.C., and J.E.W. wrote the manuscript with input from the other authors.

\section{References}

Albrecht TR, Shevtsov SP, Wu Y, Mascibroda LG, Peart NJ, Huang KL, Sawyer IA, Tong L, Dundr M, Wagner EJ. 2018. Integrator subunit 4 is a 'Symplekin-like' scaffold that associates with INTS9/11 to form the Integrator cleavage module. Nucleic Acids Res 46: 4241-4255. doi:10.1093/nar/gky100

Arndt KM, Reines D. 2015. Termination of transcription of short noncoding RNAs by RNA polymerase II. Annu Rev Biochem 84: 381-404. doi:10.1146/annurev-biochem-060614-034457

Baillat D, Wagner EJ. 2015. Integrator: surprisingly diverse functions in gene expression. Trends Biochem Sci 40: 257-264. doi:10.1016/j.tibs.2015.03.005

Baillat D, Hakimi MA, Näär AM, Shilatifard A, Cooch N, Shiekhattar R. 2005. Integrator, a multiprotein mediator of small nuclear RNA processing, associates with the C-terminal re- peat of RNA polymerase II. Cell 123: 265-276. doi:10.1016/j .cell.2005.08.019

Barbieri E, Trizzino M, Welsh SA, Owens TA, Calabretta B, Carroll M, Sarma K, Gardini A. 2018. Targeted enhancer activation by a subunit of the integrator complex. Mol Cell 71: 103-116.e7. doi:10.1016/j.molcel.2018.05.031

Belotserkovskii BP, Soo Shin JH, Hanawalt PC. 2017. Strong transcription blockage mediated by R-loop formation within a Grich homopurine-homopyrimidine sequence localized in the vicinity of the promoter. Nucleic Acids Res 45: 6589-6599. doi:10.1093/nar/gkx403

Berg MG, Singh LN, Younis I, Liu Q, Pinto AM, Kaida D, Zhang Z, Cho S, Sherrill-Mix S, Wan L, et al. 2012. U1 snRNP determines mRNA length and regulates isoform expression. Cell 150: 53-64. doi:10.1016/j.cell.2012.05.029

Brannan K, Kim H, Erickson B, Glover-Cutter K, Kim S, Fong N, Kiemele L, Hansen K, Davis R, Lykke-Andersen J, et al. 2012. mRNA decapping factors and the exonuclease Xrn2 function in widespread premature termination of RNA polymerase II transcription. Mol Cell 46: 311-324. doi:10.1016/j.molcel .2012 .03 .006

Braunschweig U, Gueroussov S, Plocik AM, Graveley BR, Blencowe BJ. 2013. Dynamic integration of splicing within gene regulatory pathways. Cell 152: 1252-1269. doi:10.1016/j.cell .2013 .02 .034

Cazalla D, Xie M, Steitz JA. 2011. A primate herpesvirus uses the integrator complex to generate viral microRNAs. Mol Cell 43: 982-992. doi:10.1016/j.molcel.2011.07.025

Chalamcharla VR, Folco HD, Dhakshnamoorthy J, Grewal SI. 2015. Conserved factor Dhp1/Rat1/Xrn2 triggers premature transcription termination and nucleates heterochromatin to promote gene silencing. Proc Natl Acad Sci 112: 1554815555.

Chen J, Ezzeddine N, Waltenspiel B, Albrecht TR, Warren WD, Marzluff WF, Wagner EJ. 2012. An RNAi screen identifies additional members of the Drosophila Integrator complex and a requirement for cyclin C/Cdk8 in snRNA $3^{\prime}$-end formation. RNA 18: 2148-2156. doi:10.1261/rna.035725.112

Chen J, Waltenspiel B, Warren WD, Wagner EJ. 2013. Functional analysis of the integrator subunit 12 identifies a microdomain that mediates activation of the Drosophila integrator complex. I Biol Chem 288: 4867-4877. doi:10.1074/jbc.M112 .425892

Chiu AC, Suzuki HI, Wu X, Mahat DB, Kriz AJ, Sharp PA. 2018. Transcriptional pause sites delineate stable nucleosome-associated premature polyadenylation suppressed by U1 snRNP. Mol Cell 69: 648-663.e7. doi:10.1016/j.molcel.2018.01.006

Connelly S, Manley JL. 1988. A functional mRNA polyadenylation signal is required for transcription termination by RNA polymerase II. Genes Dev 2: 440-452. doi:10.1101/gad.2.4.440

Core L, Adelman K. 2019. Promoter-proximal pausing of RNA polymerase II: a nexus of gene regulation. Genes Dev 33: 960982. doi:10.1101/gad.325142.119

Crossley MP, Bocek M, Cimprich KA. 2019. R-Loops as cellular regulators and genomic threats. Mol Cell 73: 398-411. doi:10 .1016/j.molcel.2019.01.024

Dower K, Kuperwasser N, Merrikh H, Rosbash M. 2004. A synthetic A tail rescues yeast nuclear accumulation of a ribozyme-terminated transcript. RNA 10: 1888-1899. doi:10 $.1261 /$ rna.7166704

Eaton JD, Davidson L, Bauer DLV, Natsume T, Kanemaki MT, West S. 2018. Xrn2 accelerates termination by RNA polymerase II, which is underpinned by CPSF73 activity. Genes Dev 32: 127-139. doi:10.1101/gad.308528.117 
Ezzeddine N, Chen J, Waltenspiel B, Burch B, Albrecht T, Zhuo M, Warren WD, Marzluff WF, Wagner EJ. 2011. A subset of Drosophila integrator proteins is essential for efficient U7 snRNA and spliceosomal snRNA 3'-end formation. Mol Cell Biol 31: 328-341. doi:10.1128/MCB.00943-10

Festa RA, Thiele DJ. 2011. Copper: an essential metal in biology. Curr Biol 21: R877-R883. doi:10.1016/j.cub.2011.09.040

Fong N, Brannan K, Erickson B, Kim H, Cortazar MA, Sheridan RM, Nguyen T, Karp S, Bentley DL. 2015. Effects of transcription elongation rate and Xrn2 exonuclease activity on RNA polymerase II termination suggest widespread kinetic competition. Mol Cell 60: 256-267. doi:10.1016/j.molcel.2015.09 .026

Fury MG, Zieve GW. 1996. U6 snRNA maturation and stability. Exp Cell Res 228: 160-163. doi:10.1006/excr.1996.0311

Gardini A, Baillat D, Cesaroni M, Hu D, Marinis JM, Wagner EJ, Lazar MA, Shilatifard A, Shiekhattar R. 2014. Integrator regulates transcriptional initiation and pause release following activation. Mol Cell 56: 128-139. doi:10.1016/j.molcel.2014.08 .004

Gómez-Orte E, Sáenz-Narciso B, Zheleva A, Ezcurra B, de Toro M, López R, Gastaca I, Nilsen H, Sacristán MP, Schnabel $\mathrm{R}$, et al. 2019. Disruption of the Caenorhabditis elegans Integrator complex triggers a non-conventional transcriptional mechanism beyond snRNA genes. PLoS Genet 15: e1007981. doi:10.1371/journal.pgen.1007981

Günther V, Lindert U, Schaffner W. 2012. The taste of heavy metals: gene regulation by MTF-1. Biochim Biophys Acta 1823: 1416-1425. doi:10.1016/j.bbamcr.2012.01.005

Hernandez N. 1985. Formation of the $3^{\prime}$ end of U1 snRNA is directed by a conserved sequence located downstream of the coding region. EMBO I 4: 1827-1837. doi:10.1002/j.14602075.1985.tb03857.x

Kaida D, Berg MG, Younis I, Kasim M, Singh LN, Wan L, Dreyfuss G. 2010. U1 snRNP protects pre-mRNAs from premature cleavage and polyadenylation. Nature 468: 664-668. doi:10 $.1038 /$ nature09479

Kamieniarz-Gdula K, Proudfoot NJ. 2019. Transcriptional control by premature termination: a forgotten mechanism. Trends Genet 35: 553-564. doi:10.1016/j.tig.2019.05.005

Kamieniarz-Gdula K, Gdula MR, Panser K, Nojima T, Monks J, Wiśniewski JR, Riepsaame J, Brockdorff N, Pauli A, Proudfoot NJ. 2019. Selective roles of vertebrate PCF11 in premature and full-length transcript termination. Mol Cell 74: 158-172.e9. doi:10.1016/j.molcel.2019.01.027

Kapranov P, Cheng J, Dike S, Nix DA, Duttagupta R, Willingham AT, Stadler PF, Hertel J, Hackermuller J, Hofacker IL, et al. 2007. RNA maps reveal new RNA classes and a possible function for pervasive transcription. Science 316: 1484-1488. doi:10.1126/science.1138341

Kramer MC, Liang D, Tatomer DC, Gold B, March ZM, Cherry S, Wilusz JE. 2015. Combinatorial control of Drosophila circular RNA expression by intronic repeats, hnRNPs, and SR proteins. Genes Dev 29: 2168-2182. doi:10.1101/gad.270421.115

LaCava J, Houseley J, Saveanu C, Petfalski E, Thompson E, Jacquier A, Tollervey D. 2005. RNA degradation by the exosome is promoted by a nuclear polyadenylation complex. Cell 121: 713-724. doi:10.1016/j.cell.2005.04.029

Lai F, Gardini A, Zhang A, Shiekhattar R. 2015. Integrator mediates the biogenesis of enhancer RNAs. Nature 525: 399-403. doi:10.1038/nature14906

Lubas M, Christensen MS, Kristiansen MS, Domanski M, Falkenby LG, Lykke-Andersen S, Andersen JS, Dziembowski A, Jensen TH. 2011. Interaction profiling identifies the human nuclear exosome targeting complex. Mol Cell 43: 624-637. doi:10.1016/j.molcel.2011.06.028

Marr MT II, Isogai Y, Wright KJ, Tjian R. 2006. Coactivator crosstalk specifies transcriptional output. Genes Dev 20: 14581469. doi:10.1101/gad.1418806

Mayer A, Landry HM, Churchman LS. 2017. Pause \& go: from the discovery of RNA polymerase pausing to its functional implications. Curr Opin Cell Biol 46: 72-80. doi:10.1016/j.ceb.2017 .03 .002

Naville M, Gautheret D. 2010. Transcription attenuation in bacteria: theme and variations. Brief Funct Genomics 9: 178-189. doi:10.1093/bfgp/elq008

Nojima T, Tellier M, Foxwell J, de Almeida CR, Tan-Wong SM, Dhir S, Dujardin G, Dhir A, Murphy S, Proudfoot NJ. 2018. Deregulated expression of mammalian lncRNA through loss of SPT6 induces R-Loop formation, replication stress, and cellular senescence. Mol Cell 72: 970-984.e7. doi:10.1016/j .molcel.2018.10.011

O'Reilly D, Kuznetsova OV, Laitem C, Zaborowska J, Dienstbier M, Murphy S. 2014. Human snRNA genes use polyadenylation factors to promote efficient transcription termination. Nucleic Acids Res 42: 264-275. doi:10.1093/nar/gkt892

Porrua O, Libri D. 2015. Transcription termination and the control of the transcriptome: why, where and how to stop. Nat Rev Mol Cell Biol 16: 190-202. doi:10.1038/nrm3943

Rubtsova MP, Vasilkova DP, Moshareva MA, Malyavko AN, Meerson MB, Zatsepin TS, Naraykina YV, Beletsky AV, Ravin NV, Dontsova OA. 2019. Integrator is a key component of human telomerase RNA biogenesis. Sci Rep 9: 1701. doi:10 .1038/s41598-018-38297-6

Shah N, Maqbool MA, Yahia Y, El Aabidine AZ, Esnault C, Forné I, Decker TM, Martin D, Schüller R, Krebs S, et al. 2018. Tyrosine-1 of RNA Polymerase II CTD controls global termination of gene transcription in mammals. Mol Cell 69: 4861.e6. doi:10.1016/j.molcel.2017.12.009

Shao W, Zeitlinger J. 2017. Paused RNA polymerase II inhibits new transcriptional initiation. Nat Genet 49: 1045-1051. doi:10.1038/ng.3867

Skaar JR, Ferris AL, Wu X, Saraf A, Khanna KK, Florens L, Washburn MP, Hughes SH, Pagano M. 2015. The Integrator complex controls the termination of transcription at diverse classes of gene targets. Cell Res 25: 288-305. doi:10.1038/cr .2015 .19

Smirnova IV, Bittel DC, Ravindra R, Jiang H, Andrews GK. 2000. Zinc and cadmium can promote rapid nuclear translocation of metal response element-binding transcription factor-1. I Biol Chem 275: 9377-9384. doi:10.1074/jbc.275.13.9377

Stadelmayer B, Micas G, Gamot A, Martin P, Malirat N, Koval S, Raffel R, Sobhian B, Severac D, Rialle S, et al. 2014. Integrator complex regulates NELF-mediated RNA polymerase II pause/ release and processivity at coding genes. Nat Commun 5: 5531. doi:10.1038/ncomms6531

Tatomer DC, Liang D, Wilusz JE. 2017. Inducible expression of eukaryotic circular RNAs from plasmids. Methods Mol Biol 1648: 143-154. doi:10.1007/978-1-4939-7204-3_11

Tian B, Manley JL. 2017. Alternative polyadenylation of mRNA precursors. Nat Rev Mol Cell Biol 18: 18-30. doi:10.1038/ nrm.2016.116

Venters CC, Oh JM, Di C, So BR, Dreyfuss G. 2019. U1 snRNP Telescripting: suppression of premature transcription termination in introns as a new layer of gene regulation. Cold Spring Harb Perspect Biol 11: a032235. doi:10.1101/cshperspect .a032235 
Vihervaara A, Duarte FM, Lis JT. 2018. Molecular mechanisms driving transcriptional stress responses. Nat Rev Genet 19: 385-397. doi:10.1038/s41576-018-0001-6

Wagschal A, Rousset E, Basavarajaiah P, Contreras X, Harwig A, Laurent-Chabalier S, Nakamura M, Chen X, Zhang K, Meziane O, et al. 2012. Microprocessor, Setx, Xrn2, and Rrp6 co-operate to induce premature termination of transcription by RNAPII. Cell 150: 1147-1157. doi:10.1016/j.cell.2012.08.004

Wilusz JE, InBaptiste CK, Lu LY, Kuhn CD, Joshua-Tor L, Sharp PA. 2012. A triple helix stabilizes the $3^{\prime}$ ends of long noncoding RNAs that lack poly(A) tails. Genes Dev 26: 2392-2407. doi:10.1101/gad.204438.112

Wu Y, Albrecht TR, Baillat D, Wagner EJ, Tong L. 2017. Molecular basis for the interaction between Integrator subunits IntS9 and IntS11 and its functional importance. Proc Natl Acad Sci 114: 4394-4399. doi:10.1073/pnas.1616605114

Xie M, Zhang W, Shu MD, Xu A, Lenis DA, DiMaio D, Steitz JA. 2015. The host Integrator complex acts in transcription-inde- pendent maturation of herpesvirus microRNA 3' ends. Genes Dev 29: 1552-1564. doi:10.1101/gad.266973.115

Xu Z, Wei W, Gagneur J, Perocchi F, Clauder-Münster S, Camblong J, Guffanti E, Stutz F, Huber W, Steinmetz LM. 2009. Bidirectional promoters generate pervasive transcription in yeast. Nature 457: 1033-1037. doi:10.1038/nature 07728

Yang XC, Sullivan KD, Marzluff WF, Dominski Z. 2009. Studies of the $5^{\prime}$ exonuclease and endonuclease activities of CPSF-73 in histone pre-mRNA processing. Mol Cell Biol 29: 31-42. doi:10.1128/MCB.00776-08

Zinder JC, Lima CD. 2017. Targeting RNA for processing or destruction by the eukaryotic RNA exosome and its cofactors. Genes Dev 31: 88-100. doi:10.1101/gad.294769.116

Zybailov B, Mosley AL, Sardiu ME, Coleman MK, Florens L, Washburn MP. 2006. Statistical analysis of membrane proteome expression changes in Saccharomyces cerevisiae. I Proteome Res 5: 2339-2347. doi:10.1021/pr060161n 


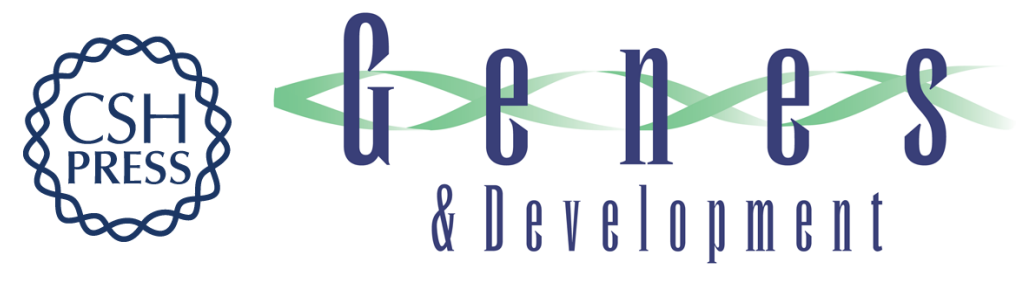

\title{
The Integrator complex cleaves nascent mRNAs to attenuate transcription
}

\author{
Deirdre C. Tatomer, Nathan D. Elrod, Dongming Liang, et al.
}

Genes Dev. 2019, 33: originally published online September 17, 2019

Access the most recent version at doi:10.1101/gad.330167.119

\section{Supplemental http://genesdev.cshlp.org/content/suppl/2019/09/17/gad.330167.119.DC1 Material}

References This article cites 59 articles, 18 of which can be accessed free at:

http://genesdev.cshlp.org/content/33/21-22/1525.full.html\#ref-list-1

Creative This article is distributed exclusively by Cold Spring Harbor Laboratory Press for the first Commons six months after the full-issue publication date (see

License http://genesdev.cshlp.org/site/misc/terms.xhtml). After six months, it is available under a Creative Commons License (Attribution-NonCommercial 4.0 International), as described at http://creativecommons.org/licenses/by-nc/4.0/.

Email Alerting Receive free email alerts when new articles cite this article - sign up in the box at the top Service right corner of the article or click here.

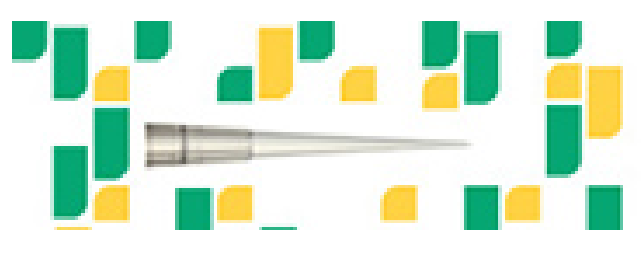

Focused on your science. 